\title{
PTSD plus depression are comorbid conditions but depression can occur independently in the acute aftermath of trauma
}

O'Donnell ML, Creamer M, Pattison P. Posttraurnatic stress disorder and depression following trauma: understanding comorbidity. Am J Psychiatr 2004;161:1390-6.

\section{Q Following trauma, are depression and post-traumatic stress disorder separate conditions, or are they a single traumatic stress construct?}

METHODS

$\square$

Design: Prospective longitudinal study

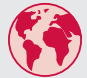

Setting: Victoria, Australia; timeframe not stated.

0

Population: 363 people aged $18-70$ years admitted to a level trauma service with injuries requiring hospital stays of $>1$ day who were stable and without pain. Exclusions included severe brain injury or recent opioid analgesia.

(1)

Assessment: The relations between post-traumatic stress disorder (PTSD), depression, and predictor variables were examined using canonical correlations and multinomial logistic regression.

Outcomes: PTSD was assessed with the Clinician-Administered PTSD Scale (DSM-IV). Participants were subsyndromal if they met re-experiencing symptoms and either avoidance or arousa symptoms criteria. Depression and minor depression were diagnosed with the Structured Clinical Interview (DSM-IV). People in the comorbid PTSD/depression category met full or subsyndrome criteria for both. Trauma characteristics, including admission to intensive care and length of stay were recorded, as was injury severity (using the Injury Severity Score). Assessments were performed by telephone at hospital discharge (mean of

8 days after injury), and by telephone at 3 months, and 12 months.

Follow up period: 12 months.

\section{MAIN RESULTS}

At 3 months, 15\% of participants met full criteria for PTSD, depression, or both $(21 \%$ including those meeting subsyndromal criteria). At 12 months, $14 \%$ of individuals met full criteria for PTSD, depression, or both $(21 \%$ including those meeting subsyndromal criteria). $63 \%$ of people with symptoms of PTSD at 3 months had PTSD, depression, or comorbid illness at 12 months, compared with $60 \%$ for those with comorbid illness at 3 months. In contrast, $92 \%$ of those identified as having depression at 3 months had no diagnosis after 1 year, suggesting characteristics of depression at 3 months that differentiate it from PTSD syndromes.

For correspondence: Dr O'Donnell, Australian Centre for Posttraumatic Mental Health, ARMC Repat Campus, Waterdale Rd., Heidelberg, Victoria 3081, Australia; mod@unimelb.edu.au

Sources of funding: Victorian Trauma Foundation.

\section{CONCLUSIONS}

Comorbid PTSD/depression is virtually indistinguishable from PTSD; however, in the acute but not chronic phase, depression does appear to be a separate construct, with a better prognosis.

\section{NOTES}

Of 363 initial participants, 337 completed the 3 month follow up, 307 completed the 12 month follow up, and 301 completed both.

\section{Commentary}

7 his study examines the longitudinal course and risk factors of depressive and post-traumatic stress disorder (PTSD) symptoms in a group of 363 injury survivors admitted to a trauma centre. ${ }^{1}$ All participants received a comprehensive evaluation while in hospital and were interviewed by telephone three and 12 months after their initial assessment. Three hundred and one participated in evaluations at all three points. Seventy four per cent had been in motor vehicle accidents and $56 \%$ had incurred mild traumatic brain injury. Because this study is centred on accidental trauma victims, the findings cannot be generalised to survivors of rape, assault, terrorism, warfare, or natural and manmade disasters. In such traumas other factors may influence the risks for, and course of, psychiatric conditions.

The study provides a very thoughtful and precise examination of psychiatric symptoms in trauma survivors across time. Importantly, it included examination of those with subsyndromal conditions as well as those who met full criteria for a psychiatric condition. Sixty nine per cent of patients remained free of either a full or subsyndromal diagnosis across time and $49 \%$ of those with a condition (full or subsyndromal) at three months no longer had a diagnosis at 12 months. While a majority of those with PTSD, or PTSD and depression at three months continued to have a psychiatric disorder at 12 months, $92 \%$ of those with depression alone at three months had no diagnosis at 12 months.

The study also presents an analysis supporting the theory that PTSD alone and PTSD with depression may be the result of a mutual set of patient and trauma characteristics, while depression alone is perhaps mediated by other factors.

When assessing trauma victims, it is important to recognise that most will do well with the passage of time. Those with depression alone have an excellent prognosis and should respond to routine clinical practices. Those with symptoms of PTSD alone or PTSD and depression have a more guarded course, with the majority meeting criteria for one or another condition at 12 months.

Thomas A Grieger, MD Associate Professor of Psychiatry, Uniformed Services University of the Health Sciences, Bethesda, MD, USA

1 O'Donnell M, Creamer M, Pattison P, et al. Psychiatric morbidity following injury. Am J Psychiatry 2004;161:507-14. 